\title{
Necesidad de adaptar los procesos de comunicación en salud a la Sociedad Red. Nuevas formas relacionales entre profesional sanitario y paciente.
}

\author{
Rosa María TORRES VALDÉS \\ Universidad de Alicante (España) \\ rosa.torres@ua.es \\ Alba Santa Soriano \\ Universidad de Alicante (España) \\ alba.santa@ua.es
}

\begin{abstract}
Resumen
La comunicación terapéutica y la relación de ayuda entre profesionales sanitarios y pacientes, requiere de procesos de adaptación al nuevo escenario de la denominada e-Salud, lo que no supone el abandono de la comunicación interpersonal y relación humana directa, sino que implica aprovechar el potencial de las TIC, ampliando con ello las posibilidades de interacción social que permiten promover el empoderamiento para la salud del ciudadano. El artículo presenta un estudio de carácter exploratorio basado en la revisión documental, con estudio de casos, que permita identificar necesidades sentidas y percibidas sobre la comunicación en salud y su adaptación a la Sociedad Red, atendiendo específicamente a las relaciones profesionales sanitarios-pacientes que emergen en este nuevo contexto de la e-Salud.
\end{abstract}

Palabras clave: Necesidades sentidas, necesidades percibidas, e-salud, sociedad red, comunicación terapéutica, profesional sanitario, paciente, TIC.

Need to adapt health communication processes to relational Network Society. New relational forms between healthcare professionals and patients.

\begin{abstract}
Therapeutic communication and aid relationship between healthcare professionals and patients requires adaptation process to the new scenario of the so-called e- Health, which does not involve the rejection of interpersonal communication and direct human relationship. It involves harnessing the potential ICT, thus expanding the possibilities of social interaction that helps to promote the citizen health empowerment. This paper presents an exploratory study based on literature review, with case studies, to identify felt needs and perceived needs on health communication and its adaptation to Network Society, taking in consideration primarily for professional and patient relationships emerging in this new context of e-Health.
\end{abstract}

Key words: Felt needs, perceived needs, e- health, network society, therapeutic communication, healthcare, patient, ICT.

\section{Referencia normalizada:}

Torres Valdés, R. M. y Santa Soriano, A. (2013) Necesidad de adaptar los procesos de comunicación en salud a la Sociedad Red. Nuevas formas relacionales entre profesional sanitario y paciente. Historia y Comunicación Social. Vol. 18. № Especial Noviembre. Págs. 651-661. 
Sumario: 1. Introducción al estado de la cuestión. 2. Objetivos. 3. Materiales y métodos. 4. Resultados. 5. Conclusiones. 6. Referencias.

\section{Introducción al estado de la cuestión}

El concepto de Sociedad Red va calando cada vez más en todas las esferas de la vida de las personas, afectando tanto a los roles personales como profesionales. La mirada retrospectiva a la noción original de red social revela que tal expresión ha sido objeto de estudio por parte de numerosas ramas del saber. Alfred Radcliffe-Brown y John Barnes desde una aproximación antropológica, plantearon el concepto de red social como "una estructura social formada por personas o entidades conectadas y unidas entre sí por algún tipo de relación o interés común" (Ponce, 2012). Términos que hoy, en tiempos de revolución tecnológica, continúan siendo empleados en esencia por autores al referirse a las redes sociales. Por su parte, Manuel Castells, en su concepción del paradigma de la Sociedad Red como estructura social conformada por redes de información propulsadas por las Tecnologías de la Información y la Comunicación (TIC), define el concepto de Red como un conjunto de nodos interconectados, que son propulsados por el individuo a partir de intereses comunes y conectados a través de las TIC, gracias, entre otros factores, a la posibilidad de establecer modelos de intercambio de información y comunicación bidireccionales, horizontales y distribuidos (Torres \& Santa. 2011:50).

La cuestión de Salud y relación profesional sanitario-paciente no es ajena a ninguna de las definiciones de red social citadas. Así, el Glosario de Promoción de la Salud confeccionado por la Organización Mundial de la Salud (OMS) en Yakarta (Indonesia, 1997), define el término red social como aquellas "relaciones y vínculos sociales entre las personas que pueden facilitar el acceso o movilización del soporte social a favor de su salud" (Nutbeam, 1998: 30). Desde el problema de estudio que nos ocupa, esta definición podría ampliarse añadiendo al soporte social ${ }^{1}$ definido por la OMS, el soporte tecnológico de la Sociedad Red.

Por otra parte, "las aplicaciones de las TIC en el ámbito sanitario están llamadas a revolucionar la manera en que interactuamos con el Sistema, y el aspecto que éste muestra al ciudadano" (Narváez \& Toribio, 2006:5), lo que hace conveniente ahondar en la visión global que se entiende por e-Salud, prestando especial atención al impacto transformador que ello motiva para las relaciones profesionales sanitarios-pacientes, puesto que las TIC "facilitan un nuevo medio de acceso de los pacientes a sus médicos, de los profesionales entre sí y, de manera más amplia, entre todos los actores que intervienen en el sistema sanitario" (Mayer \& Leis, 2006).

Encontramos diversas definiciones de e-Salud en la literatura, propuestas por organismos relevantes en la cuestión (Ministerial Declaration, eHealth 2003; Unión Europea, 2003; L. Androuchko, ITU-D, ITU Workshop on Standardisation on eHealth, 2003; Journal of Medical Internet Research, 2001); en su conjunto, aluden a una 
característica fundamental: "la e-Salud trata de situar al ciudadano en el centro del Sistema con el fin de simplificar y mejorar su interacción con el amplio abanico de profesionales al cuidado de su salud" (Narváez \& Toribio, 2006:11). Un hecho que favorece, por consiguiente, "el cambio del actual paradigma centrado en las instituciones por un modelo centrado en los pacientes" (Jiménez, García \& Bèss, 2009 art.) y posibilita la oportunidad de redefinir el papel que juegan los actores implicados. Por citar ejemplos, se ha demostrado que un uso eficiente de las TIC en la sanidad permite transformar el rol del paciente, asumiendo éste "un papel cada vez más responsable y activo en el cuidado de su salud", y replantear el papel del profesional sanitario como facilitador, "que deberá centrarse en el fortalecimiento de la persona para que domine, aún más, las competencias comunicativas y persuasivas, al mismo tiempo que (...) enfatizar la imagen de presencia constante del profesional y de acceso continuo del usuario a la intervención" (Hernández \& Gómez-Zúñiga, 2007: 2).

En el caso español, la adopción de la e-Salud en el sistema sanitario se ha articulado a través del programa "Sanidad en Línea", recogido en el Plan Avanza (20052010). Aún cuando éste comienza con una afirmación ambiciosa, "Avanzar hacia la Sociedad en Red, ya no es una opción" (Red.es, 2005: 9) sus medidas se limitan a la implantación de cuatro nuevas aplicaciones y contenidos en el ámbito sanitario: tarjeta sanitaria, historia clínica digital, receta electrónica y nodo de intercambio Sistema Nacional de Salud.

Se vislumbran retos de elevada trascendencia social que, a su vez, plantean importantes desafíos a los profesionales sanitarios, gestores y políticos con competencias en sanidad; evidenciando su responsabilidad pública en la gestión de riesgos ligados, por ejemplo, a una "posible mala calidad de la información, fraudes y falta de respeto a la intimidad de las personas u otros derechos de los usuarios" (Carnicero, 2006:21), y que han de ser tenidos muy en cuenta en una nueva articulación de relaciones profesionales sanitarios-pacientes mediadas por la tecnología.

Es más, estas situaciones de riesgo pueden ser consideradas como un síntoma que alerta sobre el ritmo insuficiente de actualización tecnológica del sistema sanitario para garantizar su eficiencia y capacidad de dar respuesta competitiva a las necesidades sentidas y percibidas de pacientes y profesionales sanitarios, manifestadas en las conclusiones del estudio de prospectiva "e-Salud 2020" (Narváez \& Toribio, 2006). Cada vez son más los ciudadanos que comienzan a utilizar aplicaciones móviles independientes o consultar fuentes de información sanitarias en Internet no reguladas para dar respuesta a sus necesidades e inquietudes sobre una dolencia o enfermedad. Según datos del observatorio español ONTSI, publicados en el estudio "Los ciudadanos ante la e-Sanidad" $(2012)^{2}$, Internet se posiciona como una de las principales fuentes de información en salud para los ciudadanos, donde "el 59\% del tráfico de visitas va dirigido a webs privadas", y cada vez son más los ciudadanos que utilizan las redes sociales para estos fines. De ellos, "el 36,6\% de los usuarios deposita bastante o mucha confianza en la información sanitaria que encuentra en las redes sociales", y en cuanto a su valor útil, "el $38,9 \%$ de los usuarios considera que éstas le 
ayudan en la aclaración de dudas" y un " $26,1 \%$ las utiliza para conocer y compartir experiencias sobre un determinado problema de salud".

Por último, atendiendo al problema de estudio planteado, resulta conveniente revisar la evolución de las formas de relación entre profesionales sanitarios y pacientes en el nuevo escenario de la e-Salud, desde la comunicación interpersonal hasta la comunicación mediada por la tecnología, desde comunidades físicas de afectados por una patología a comunidades virtuales de apoyo de los mismos. No sin antes establecer claramente a qué tipo de comunicación en salud se hace referencia en este trabajo.

Es preciso, por tanto, señalar que cuando hablamos de relación personal sanitario-paciente (especialmente éste último) nos estamos refiriendo específicamente a la comunicación terapéutica y relación de ayuda. Así, éstas constituyen el objeto de estudio que abordamos en el trabajo de investigación que se presenta estructurado en siete apartados: introducción para esbozar el estado de la cuestión, definiciones operativas relevantes para facilitar la comprensión del tema elegido, objetivos de estudio, materiales y métodos, resultados y conclusiones; así como referencias bibliográficas que han sido consultadas.

\subsection{Definiciones operativas}

\subsubsection{Relación profesional sanitario-paciente.}

"Es la interacción que se establece entre el médico y el paciente durante el curso de la enfermedad (aguda o crónica). Es una relación interpersonal de tipo profesional que sirve de base a la gestión de salud. Esta relación puede influir en el curso de la enfermedad y en la eficacia del tratamiento" (Pérez, Vidaillet, Carnot \& Duane, 2003).

\subsubsection{Comunicación terapéutica.}

Proceso mediante el cual el profesional sanitario establece una relación con el paciente (...) basada en la "confianza y ayuda, en la que haya disposición de diálogo y escucha, utilizando una terminología clara y comprensible que facilite la comunicación y que permita identificar y satisfacer las necesidades del paciente de forma asertiva y oportuna" (Naranjo \& Ricaurte, 2006: 1). Implica "fomentar las relaciones interpersonales positivas (...) y entraña un lenguaje verbal y no verbal" (Navarro, Rivas \& Villafranca, 2008: 7).

\subsubsection{Relación de ayuda.}

Es la interacción que se da entre el profesional sanitario y el paciente mediante la comunicación terapéutica (Navarro, Rivas \& Villafranca, 2008: 15). 


\subsubsection{Necesidades sentidas y percibidas.}

"Berwick (1989:55) hace una clara distinción entre las necesidades percibidas y las necesidades sentidas" (Del Campo, 2004:11), según la cual podemos entender las necesidades sentidas como aquellas que tienen los públicos (pacientes) y necesidades percibidas como aquellas que tiene la organización (profesionales sanitarios e instituciones). Su identificación, contraste y conexión son la base de una gestión relacional exitosa entre profesionales sanitarios-pacientes, puesto que

"la percepción que finalmente tenga el sistema social en su conjunto sobre si las políticas de desarrollo están diseñadas y ejecutadas para ir al encuentro con las necesidades sentidas por cada uno de los subconjuntos o colectividades del sistema, dependerá de la calidad de los procesos puestos en marcha a la hora de concebir, planificar y ejecutar dichas políticas" (Torres, 2008: 146).

\subsubsection{Empoderamiento para la salud.}

Según el Glosario de Promoción de la Salud de la OMS, se define como el "proceso social, cultural, psicológico o político" mediante el cual "las personas adquieren un mayor control sobre las decisiones y acciones que afectan a su salud", siendo individuos y grupos sociales "capaces de expresar sus necesidades, plantear sus preocupaciones, diseñar estrategias de participación en la toma de decisiones y llevar a cabo acciones políticas, sociales y culturales para hacer frente a sus necesidades" (Nutbeam, 1998:16).

\section{Objetivos}

El objetivo general de la investigación es identificar necesidades sentidas y percibidas sobre la comunicación en salud y su adaptación a la Sociedad Red en el marco de las relaciones profesionales sanitarios-pacientes emergentes en e-Salud.

Para ello, los objetivos específicos propuestos son: establecer un paralelismo entre la definición de Redes sociales para la salud de la OMS, y Sociedad Red de Manuel Castells; conocer el grado de utilización y tipo de uso de las TIC por parte de los profesionales sanitarios en relación con los pacientes; conocer el grado de utilización y tipo de uso de las TIC por parte de los pacientes en relación con su dolencia y profesional que le atiende, $\mathrm{y}$, finalmente, conocer cuáles son las barreras, oportunidades y factores de éxito para transformar la comunicación en salud y adaptarnos a la Sociedad Red. 


\section{Materiales y métodos}

El método de estudio empleado en este trabajo ha sido de carácter exploratorio, fundamentado en técnicas de revisión documental sobre directrices, conceptos y estudio de casos, extraídos de documentos bibliográficos librados por organizaciones pertinentes, prestando especial interés a aspectos como: percepciones, creencias y actitudes de los actores partícipes, información de salud en Internet, tipos de interacción interpersonal establecidos, instrumentos y medios tecnológicos utilizados, habilidades comunicativas y persuasivas, experiencia del paciente, capacidad empática y de motivación del profesional sanitario, así como posibles objetivos de comunicación logrados e identificación de necesidades sentidas y percibidas por las partes.

El trabajo recurre al estudio de casos, específicamente a tres experiencias que han sido desarrolladas en el marco de la e-Salud. Los criterios de elegibilidad han sido: constituir un ejemplo de aplicación concreto y diferente en e-Salud y prestar especial atención a la descripción de métodos de comunicación y relación. Se pretende con el análisis de los casos elegidos, describir las peculiaridades de las relaciones profesionales sanitarios-pacientes que en cada uno de los casos acontece, presentando especial atención al grado de utilización y tipo de uso que los actores hacen de las TIC, así como a barreras, oportunidades y factores de éxito destacados. Para ello, en el apartado de Resultados se mostrará un análisis comparativo de aspectos como: roles jugados por los actores, objetivos de comunicación pretendidos, tipos de relación establecidas, medios e instrumentos tecnológicos empleados, gestión de riesgos en juego y posibles necesidades sentidas y percibidas tratadas en el caso.

Los casos propuestos para estudio son:

a) Universidad del Paciente (http://www.universidadpacientes.org/), promovida por la Universidad Autónoma de Barcelona y la Fundació Biblioteca Josep Laporte como iniciativa originaria del Foro Español de los Pacientes (FEP).

b) Hospital Virtual, sistema de telemedicina para la atención integral de pacientes con VIH/SIDA en su domicilio a través de Internet, promovido por el Hospital Clínico de Barcelona.

c) Distintivo AppSaludable (http://www.calidadappsalud.com/), promovido por la Agencia de Calidad Sanitaria de Andalucía. Es un ejemplo de regulación institucional, a modo de certificación, de aplicaciones móviles.

\section{Resultados}

A continuación, se presentan los resultados extraídos de un análisis comparativo del estudio de casos abordado anteriormente, atendiendo al modelo de comunicación terapéutica y relación de ayuda que acontece en cada caso. El objetivo es ayudar a extraer conclusiones sobre el problema de estudio propuesto, prestando especial 
atención a los siguientes aspectos: roles jugados por los profesionales sanitarios (PS) y pacientes $(\mathrm{P})$, objetivos de comunicación pretendidos (pudiendo ser: informacionales, motivacionales o de comportamiento), tipos de relación establecidas, medios e instrumentos tecnológicos empleados, gestión de riesgos planteados y posibles necesidades sentidas y percibidas de los actores.

\section{Tabla 1}

\begin{tabular}{|c|c|c|c|}
\hline & $\begin{array}{l}\text { Universidad del } \\
\text { paciente }\end{array}$ & Hospital virtual & Aplicaciones móviles \\
\hline Roles actores & $\begin{array}{l}\text { PS: facilitador, } \\
\text { prescriptor, abogacía por } \\
\text { la salud } \\
\text { P: participante activo, } \\
\text { abogacía por la salud. }\end{array}$ & $\begin{array}{l}\text { PS: profesional, facilitador, } \\
\text { prescriptor. } \\
\text { P: participante activo, } \\
\text { autogestión. }\end{array}$ & $\begin{array}{l}\text { PS: pasivo. } \\
\text { P: autogestión. }\end{array}$ \\
\hline $\begin{array}{l}\text { Objetivos de } \\
\text { comunicación }\end{array}$ & Informacionales & $\begin{array}{l}\text { Informacionales. } \\
\text { Motivacionales. } \\
\text { Comportamiento. }\end{array}$ & Comportamiento. \\
\hline $\begin{array}{l}\text { Tipos de } \\
\text { relaciones }\end{array}$ & $\begin{array}{l}\text { PS-P } \\
\text { PS-PS } \\
\text { P-P }\end{array}$ & $\begin{array}{l}\text { PS-P } \\
\text { PS-PS } \\
\text { P-P }\end{array}$ & P-P \\
\hline $\begin{array}{l}\text { Instrumentos } \\
\text { y medios } \\
\text { tecnológicos }\end{array}$ & $\begin{array}{l}\text { Página web. } \\
\text { Redes sociales. } \\
\text { Aulas virtuales. }\end{array}$ & $\begin{array}{l}\text { Página web. } \\
\text { Consultas virtuales. } \\
\text { Telefarmacía. } \\
\text { Biblioteca virtual. } \\
\text { Comunidad de apoyo. }\end{array}$ & $\begin{array}{l}\text { Página web. } \\
\text { APP. }\end{array}$ \\
\hline $\begin{array}{l}\text { Gestión de } \\
\text { riesgos }\end{array}$ & $\begin{array}{l}\text { Certificación } \\
\text { información }\end{array}$ & $\begin{array}{l}\text { Certificación información } \\
\text { Seguridad } \\
\text { Confidencialidad }\end{array}$ & Gestión privada. \\
\hline $\begin{array}{l}\text { Necesidades } \\
\text { sentidas y } \\
\text { percibidas }\end{array}$ & $\begin{array}{l}\text { PS: información sanitaria } \\
\text { de calidad, habilidades } \\
\text { comunicativas y } \\
\text { persuasivas. } \\
\text { P: información sanitaria } \\
\text { creíble y confiable, } \\
\text { comprensión casuística } \\
\text { enfermedad, experiencia } \\
\text { del paciente, comunidad } \\
\text { de apoyo, sentimiento } \\
\text { de pertenencia y } \\
\text { representación del } \\
\text { paciente en el sistema } \\
\text { público sanitario, mejora } \\
\text { de la comunicación entre } \\
\text { profesional sanitario y } \\
\text { paciente, autonomía. }\end{array}$ & 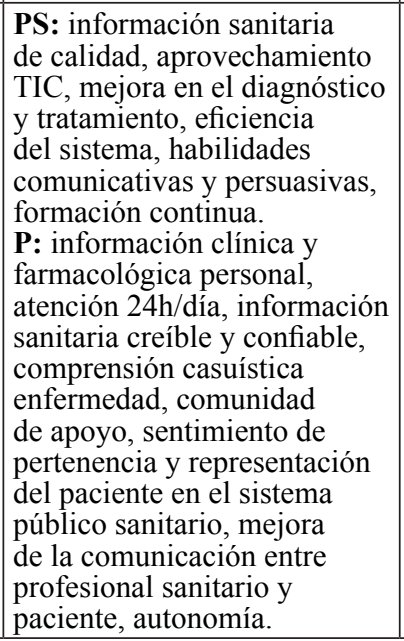 & $\begin{array}{l}\text { PS: riesgo latente } \\
\text { ante los derechos } \\
\text { de los pacientes, } \\
\text { especialmente } \\
\text { en cuanto a } \\
\text { comercialización de } \\
\text { información sensible, } \\
\text { intrusismo. } \\
\text { P: comprensión } \\
\text { casuística enfermedad, } \\
\text { autonomía. }\end{array}$ \\
\hline
\end{tabular}

Fuente: Elaboración propia. 


\title{
5. Conclusiones
}

Analizadas las numerosas iniciativas que vienen desarrollándose en el ámbito de la e-Salud, se abre la reflexión sobre los beneficios, oportunidades y retos que un uso integral de las TIC plantea a la transformación estructural del sistema sanitario. Los casos aquí analizados, son una muestra de esta realidad y nos sirven para focalizar la atención en el problema de estudio abordado y alcanzar el objetivo definido de identificar necesidades sentidas y percibidas sobre la comunicación en salud, y su adaptación a la Sociedad Red en el marco de las relaciones profesionales sanitarios-pacientes emergentes en la e-Salud.

La comunicación terapéutica y la relación de ayuda entre profesionales sanitarios y pacientes amplía sus posibilidades gracias a las TIC, especialmente con el empleo de medios e instrumentos tecnológicos que permiten una mejora sin precedentes en el acceso a información sanitaria, interacción social y relaciones entre profesionales sanitarios y pacientes, pacientes en relación con su dolencia, pacientes con otros pacientes, así como las relaciones entre los profesionales.

Posibilidades relacionales que surgen a través de las TIC, permiten "identificar preocupaciones, intereses y contrastar necesidades sentidas de los sujetos receptores con las necesidades percibidas por los sujetos promotores" (Torres, 2008: 119), trabajando desde modelos de comunicación de coorientación y velando siempre por la gestión de conflictos potenciales y la defensa de los derechos de los pacientes desde una actuación éticamente responsable.

La verdadera revolución de este panorama acontece en el componente relacional.

\begin{abstract}
"Internet ha proporcionado, a gran parte del público general y a la mayor parte de los profesionales de la salud, una oportunidad para conseguir información médica y sanitaria y recursos para la comunicación" (...) organizando sus propias redes y reconfigurando "las relaciones entre médico y paciente ante la nueva tecnología" (Katz, Rice \& Acord, 2004:13).
\end{abstract}

Estamos, pues, ante un hecho social cuya fuerza motriz son las redes sociales, una realidad manifiesta que invita a ampliar la propia concepción de redes sociales propuesta por la OMS, sumando al soporte social, el prometedor soporte tecnológico, como estrategia para transformar la comunicación en salud para adaptarnos a la Sociedad Red. Políticas y prácticas que caminan hacia la consecución de un interés común: promover el empoderamiento para la salud de los ciudadanos, y que requieren de una apuesta decidida del sistema sanitario que atienda a reducir las múltiples brechas digitales que habitan hoy en la Sociedad Red.

\section{Referencias}

ASHOKA (2009). Albert Jovell (Barcelona), Emprendedor Social de Ashoka 2009. [Documento en línea recuperado el 30/05/2013 de http://spain.ashoka.org/sites/ spainsix/files/Albert\%20Jovell.pdf]. 
BLANCH, J., CÁCERES, C., NOMDEDEU, M., ROUSAUD, A., GÓMEZ, J. \& GARCÍA, F. (2007). Telemedicina y VIH. Monográfico Intervención en Salud en la Red. UOC Papers, 4. [Documento en línea recuperado el 31/05/2013 de http:// www.uoc.edu/uocpapers/4/dt/esp/monografico.pdf]

CARNICERO, J. (2006). Informe SEIS. Luces y sombras de la información de salud en Internet. Pamplona: SEIS, Sociedad Española de Informática de la Salud. [Documento en línea recuperado el 30/05/2013 de http://www.seis.es/jsp/base. jsp?contenido $=/$ jsp/publicaciones/inforseis.jsp\&id $=5.2 \&$ informeid $=2 \&$ titulo]

DEL CAMPO, R.M. (2004). Análisis de las necesidades de los pacientes estudiantes japoneses de ELE en las universidades japonesas. Madrid: Universidad Antonio de Nebrija. [Documento en línea recuperado el 30/05/2013 de http://www.doredin.mec.es/documentos/00820103006900.pdf]

HERNÁNDEZ, E. \& GÓMEZ-ZÚÑIGA, B. (2007). Una aproximación a la intervención en salud en la red. Monográfico Intervención en Salud en la Red. UOC Papers, 4. [Documento en línea recuperado el 31/05/2013 de http://www.uoc.edu/ uocpapers/4/dt/esp/monografico.pdf]

JIMÉNEZ, J., GARCÍA, J.F. \& BESS, MB (2009). Estructura organizativa de las comunidades virtuales de salud en español: estudio exploratorio. RevistaeSalud. com, 5:17. [Documento en línea recuperado el 30/05/2013 de http://www.revistaesalud.com/index.php/revistaesalud/article/view/285/622]

KATZ, J.E., RICE, R.E. \& ACORD, S.K. (2004). Redes de salud virtual y transformaciones sociales: expectativas de centralización, experiencias de descentralización. La Sociedad Red: Una visión Global. Madrid: Alianza Editorial.

MAYER, M.A. \& LEIS, A. (2006). El correo electrónico en la relación médico-paciente: uso y recomendaciones generales. Aten Primaria, 37:7. [Documento en línea recuperado el 30/05/2013 de http://www.intramed.net/UserFiles/Files/ email1236.pdf]

NARANJO, I.C. \& RICAURTE, G.P. (2006). La comunicación con los pacientes. Investigación y Educación en Enfermería, 24:1. [Documento en línea recuperado el 30/05/2013 de http://aprendeenlinea.udea.edu.co/revistas/index.php/iee/article/ viewFile/2924/2557]

NARVÁEZ, M.I \& TORIBIO, C. (2006). Ciencias de la Salud. e-Salud 2020. Estudio de prospectiva. Madrid: Fundación OPTI y FENIN. [Documento en línea recuperado el 30/05/2013 de http://www.fenin.es/pdf/prospectiva_esalud.pdf]

NAVARRO, N., RIVAS, Z. \& VILLAFRANCA, B (2008). Comunicación terapéutica que brinda el profesional de enfermería a la paciente postaborto, en el servicio de obstetricia de la Maternidad Concepción Palacios. Caracas. [Documento en línea recuperado el 30/05/2013 de http://saber.ucv.ve/xmlui/ bitstream/123456789/1522/1/COMUNICACI\%C3\%93N\%20TERAP\%C3\%89UTICA\%20QUE\%20BRINDA\%20EL\%20PROFESIONAL\%20DE\%20 ENFERM.pdf]

NUTBEAM, D. (1998). Health promotion glossary. [Documento en línea Health Prom Intern. 13 (4): 351-3 Acceso 10 de diciembre de 2012. Recuperado el 30/05/2013 de http://www.who.int/healthpromotion/about/HPG/en/ y de http:// 
www.mecd.gob.es/dctm/ministerio/educacion/universidades/educacion-superior-universitaria/universidades- $\% 20 \% 20$ saludables/glosario.pdf?documentI$\mathrm{d}=0901 \mathrm{e} 72 \mathrm{~b} 81238071$ versión castellana p. 24-32.]

ONTSI (2012). Los Ciudadanos ante la e-Sanidad. Estudio sobre opiniones y expectativas de los ciudadanos sobre el uso y aplicación de las TIC en el ámbito sanitario. [Documento en línea recuperado el 30/05/2013 de http://www.ontsi.red.es/ ontsi/sites/default/files/informe_ciudadanos_esanidad.pdf]

PÉREZ, A., VIDAILLET, E.C., CARNOT, J. \& DUANE, O.J. (2003). La relación médico-paciente en el Sistema Nacional de Salud. Revista Cubana de Medicina General, 9:6. [Documento en línea recuperado el 30/05/2013 de http://www.bvs. sld.cu/revistas/mgi/vol19_6_03/mgi06603.htm]

PONCE, I. (2012). Monográfico Redes sociales. Ministerio de Educación, Cultura y Deporte. Observatorio Tecnológico. [Documento en línea recuperado el 30/05/2013 de http://recursostic.educacion.es/observatorio/web/es/internet/ web-20/1043-redes-sociales?start=1]

RED.ES (2005). Las TIC en el sistema nacional de Salud. El programa Sanidad en Linea. [Documento en línea recuperado el 30/05/2013 http://www.elmedicointeractivo.com/resources/files/2011/8/29/1314608362129glosario09.pdf]

TORRES, R. \& SANTA, A. (2011). Principios y técnicas de Relaciones Públicas en la estrategia de desarrollo local". Manual de estudio. [Documento en línea recuperado el 18/03/2013 de http://www.escuelagobierno.org/escuela/index. php?sub_cat $=25982]$

TORRES, $\overline{\mathrm{R}}$. (2008). Aplicación de instrumentos de Relaciones Públicas en el ámbito del Desarrollo Local. Descripción y posibilidades. Tesis doctoral. Barcelona: Universidad Autónoma de Barcelona. [Documento en línea recuperado el 30/05/2013 de http://www.tesisenred.net/handle/10803/4146]

\section{Notas}

1 Entendiendo en "Soporte social" como "aquella asistencia para las personas y los grupos desde dentro de sus comunidades que puedan servirles de ayuda para afrontar los acontecimiento y las condiciones de vida adversos y puede ofrecer un recurso positivo para mejorar la calidad de vida" siempre de la OMS a partir del trabajo de Nutbeam.

2 Datos que reproducimos por su interés para contextualizar el trabajo.

3 Definida por Nutbeam para la OMS como "una combinación de acciones individuales y sociales destinadas a conseguir compromisos políticos, apoyo para las políticas de la salud, aceptación social y apoyo de los sistemas para un determinado objetivo o programa de salud" 


\section{Las autoras}

Rosa Torres es profesora del departamento de comunicación y psicología social de la Universidad de Alicante y enseña comunicación en títulos de grado y magister en la Universidad de Alicante. Es doctora en Publicidad y Relaciones Públicas, Máster en biomedicina y biotecnología y tiene postgrado en Responsabilidad Social por RedUnirse (PNUD). Sus líneas de investigación publicaciones relacionan la comunicación, con la calidad docente, la Responsabilidad Social, Desarrollo Local, la salud $\mathrm{y}$ el fundraising.

Alba Santa es Técnico de I+D en el Observatorio Virtual de Transferencia de Tecnología de la Universidad de Alicante. Su labor se centra en divulgar las potencialidades de la vigilancia tecnológica y la inteligencia competitiva aplicada a proyectos de $\mathrm{I}+\mathrm{D}+\mathrm{i}$, desde una visión práctica de apropiación social de la tecnología y uso avanzado de Internet. Es Licenciada en Publicidad y Relaciones Públicas. 